DIPHTHERIA.

BEFORE the New York Academy of Medicine, Dr. C. E. Billington read a paper on this disease and its treatment,
which was especially valuable, as it was based entirely upon clinical and personal experience; his observations having ber of cases. The records of the Bureau of Vital Statistics
showed, said he, that in 1873 there showed, said he, that in 1873 there were over four hundred deaths from in 1875 no less than two thousand three hundred
and twenty-nine. This terrible epidemic he thought could not be checked by any therapeutic methods, but could only be stamped out by the most revolutionary and active sanitary reform. Dr. Billington has enjoyed unusual facilities for the
study of the disease, as he is one of the District Physicians study of the disease, as he is one of the District Physicians
of the Demilt Dispensary, and has seen altogether about three hundred cases, of which he has careful records of about one
half. As a result of his observation and study, he has become
fully convinced that diphtheria is a local disease, at least primarily ; and, though this is the opinion of a minority of the
authorities on the subject, he is glad to have his views corrobauthorities on the subject, he is glad to have his views corrob-
orated by such observers as Drs. Jacobi and J. Lewis Smith. orated by such observers as Drs. Jacobi and J. Lew
This conclusion is based upon the following points :
First. The local affection commences first.

Second. The gravity of the general symptom

$\mathrm{ms}$ is in proporThird. The results of treatment seem to substantiate this
Thion to the severity of the local manestations.

In the study of the nature of the disease, he said, three Inents were to be considere

(1) The contagium, which he did not propose to discuss on this occasion.
(2) The inflammation, denuding the fauces of epithelium, and resulting in membranous exudation; and system in general are, to a greater or less extent, septicæmic in character.
Dr. Billington's treatment consists mainly in local disinfection, together witl the most careful and unremitting watch-
ing and attention. The agents which he regards as most useing and attention. The agents which he regards as most use-
ful are the following, in the order in which they stand in his
estimation: Tincture of the chloride of iron, lime-water and glvcerine; and after them, salicylic and carbolic acids, sulwhich he uses in almost every case is as follows
which

$$
\begin{gathered}
\text { R-Tinct. ferri chlor., } f \text { dr. iss. } \\
\text { Glycerinæ. } \\
\text { Aquæ, aa f oz. j.-M. } \\
\text { S.-Teaspoonful every hour or half hour. }
\end{gathered}
$$

Besides being very effective, it has the merit of being pleas-
ant to the taste, which is a great desideratum for pecially when the dose has to be so frequently repeated. If the child is under two years, one drachm of the tincture of
the chloride of iron is enough, and if vomiting follows the administration of the medicine, it should not be given so often.
In connection with the above, Dr. Billington formerly em-

$$
\begin{aligned}
& \text { Potass. chlor, dr. iss. } \\
& \text { Glycerine, f oz. ss. }
\end{aligned}
$$

A teaspoonful of this was alternated with a dose of the former; so that the patient would receive one or the other
every half hour. As a substitute for the chlorate of potassium every half hour. As a substitute for the chlorat
mixtire, he now generally uses the following:

$$
\begin{aligned}
& \text { R-Acid salicylic, gr. } x-x v \text {. } \\
& \text { Sodii sulphit., gr. } \mathbf{x x}-\mathbf{x l v} \text {. } \\
& \text { Glycerinæ, f oz. ss. }
\end{aligned}
$$

Here the salicylic acid is rendered soluble by the addition of three times its weight of sulphite of sodium (borax also
has the same effect), so that in this prescription we have the advantages of both these reputed antiseptics, which are indipractical benefit. It is of great importance that in every case
in which it is practicable some sort of spray should be used
upon the throat ; and the most convenient instrument with which to accomplish this is the ordinary little perfumery spray. apparatus now in such general use. In order to annoy the child as little as possible, it is best to employ the spray immediatel
after a dose of the medicine is administered. The combin
tion generally used by Dr. Brllington is the following:

$$
\begin{aligned}
& \text { R-Acid, carbolic, m.x. } \\
& \text { Liq. calcis, } \mathrm{f} \text { oz. iv.-M }
\end{aligned}
$$

He believes that the nasal douche or syringe has saved many lives; and even when the nasal passages, apparently, the mucous membrane inaccessible to the spray. If, therethe latter, it ought to be resorted to ; and the mixture menther for the purpose.

In adults or large children it may occasionally be of to apply carefully strong tincture of iron (say two parts of membrane; but, as a rule, topical applications of caustics of astringents by the probang or camel's-lair brush do much patients from their struggles to resist, excite an increased flow of blood to the part, and really occasion further thickening and spread of the membrane. stantiated by other observers) that quinine is worse than useless in diphtheria in children; being objectionuble, if for no
other reason, on account of its bitter taste, which make other reason, on account of its
every dose dreaded by the patient.

In cases attended with high secondary fever, a full dose of to the prevalent opinion that diphtheria will never bear ant phlogistic treatment.
Dr. Billington then

mary of the cases which he had to give an interesting sum ing his statement with an allusion to the well-recognized dis advantages to be encountered in dispensary practice. Accord-
ing to his observations, about sixty-five per cent of all cases
of diphtheria occur in is quite a rare affection among adults (except in the peculiar experience of certain irregular practitioners), even when individuals are constantly and to the fullest extent exposed to the disease. He has also found that about sixty per cent of that about five per cent will prove fatal whatever plan may
be adopted. Out of one hundred and two carefully tabulated dispensary cases treated by him, fourteen died, and eighty-
eight recovered ; while of seventeen cases in private practice, e died, and sixteen recovered.

The usual duration of the attack, from the commencement the treatment to the disappearance of the diphtheritic
membranes, was only from four to six days. Twenty-four Wm. Darken, house physican to the Demilt Dispensary show. en a better result ; as not a single death occurred directly weeks after the acute attack, from some unexplained several A still later series of fourteen cases treated by Dr. Billingon in conjunction with Dr. W. E. Bullard (in order that the patients might receive the fullest possible amount of atten
ion) all recovered, so that we have fifty-five cases altogether with only one death directly attributed to the disease. In a
arge number of these the attack was of very great severity. arge number of these the attack was of very great severity.
From his observations, Dr. Billington has been induced to believe that a laryngeal complication can often be prevented or aborted by the use of the spray, and that even after the
membranes have been fully formed in this locality it is of very great service. Calomel has also proved useful in many
cases. The inhalation of hot vapor, he thinks, renders the cases. The inhalation of hot vapor, he thinks, renders the
and therefore injurious.

He did not express a positive opinion as to the identity of
coup and diphtheria, but apparently seemed to hold to the former view.-Canada Lancet.

\section{SULPHIDE OF CALCIUM.}

By T. Cortis Smith, M.D., Middleport, Ohio.

I TAKE pleasure in calling attention to this remedy for acion in a field where an agent that will produce the effects

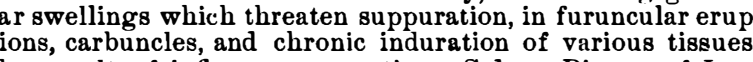
the result of inflammatory action. Sidney Ringer, of Lon-
don, has done much to bring this prominently before the pro fession as a valuable therapeutic agent. He, however, signally.
fails to give the rationale of its mode of action upon the phy. sical economy, further than to clain great alterative action for it. This is but another method of covering up our igno-
rance of the real actions of various drugs now in general
uns I liave used this agent more or less for a year past, and am
und well satisfied that it possesses some valuable properties for difficulties of the classes above named.
In cases where glandular abscesses are threatened in scrofulous children, it has the effect to hasten their removal by
either rapidly increasing their tendency to suppuration and either rapidly increasing their tendency to suppuration and
rapid healthy granulation, or of removing them quickly by diction of terms, but we may well remember that other and apparently less potent agents have similar therapeutic value. viating inflammation, and the same kind of applications are
also used to hasten auppuration. So we claim no more for also used to hasten auppuration. So we claim no more for
this agent than has long been practically claimed in these different actions for a common poultice, but we hold that it is
far more efficient. We not unfrequently meet with glandular swellingsin stru-
far more effient. mous children and adults, not of specific origin. These often Secome very large. Sometimes, after weeks of suffering and
nconvenience trom the swellings, they suppurate, and after inconvenience trom the swellings, they suppurate, and after
opening are very slow to heal, and for weeks will discharge
an unhealthy-looking pus ; and when they have healed, too an unhealthy-looking pus; and when they have healed, too
of ten leave very ugly cicatrices about the neck. If in such cases the sulphide of calcium is given, and without any other
aid, these swellings, however large they may be, will soon dis. aid, these swellings, however large they may be, will soon discase, instead of a long, tedious course of waiting through days
and nights of suffering, and increased general debility, they
will rapidly hasten to a termination by the will rapidly hasten to a termination by the suppurative
process. The pus formed under its use seems of a more audable character than that commonly observed in strumous abscesses, and after being discharged, healthy granulations of opening has closed, leaving very little trace of the former
trouble. If no pus has already formed in these large, threatening, indurated glands, then under the sulphide of calcium disappear.

Another use of no mean value for this agent is in the treat-
ment of boils. Several times in the last year have I met ment of boils. Several times in the last year have I met
with cases where a succession of crops of furuncles would appear. As one crop would commence to decline another
would put in an appearance. In all of such cases, where the would put in an appearance. In all of such cases, where the
sulphide of calcium has been resorted to, they have speedily
recovered. The boils already formed would quickly suppurate, and discharge profusely, the "core" melting away with
astonishing rapidity. The little boils just commencing to orm would rapidly disappear without ever becoming painful, and no trace of them could be observed. The points were
notably gained in the case of an old lady who was so much
annoyed from the boils that she could not sleep day or night, annoyed from the boils that she could not sleep day or night,
nor move in any direction without pain, of ten severe. In a nor move in any direction without pain, of ten severe. In a
few days she was relieved completely. Also in a recent case few days she was relieved completely. Also in a recent case
where a succession of these painful abscesses had occurred,
those present rapidly disappeared, and have not yet returned. In carbuncles I have had a more limited experience. Sid-
ney Ringer is loud in his praise of the remedy in this very ney Ringer is loud in his praise of the remedy in this very
painful affection, and cepecially mentions the rapid solution
of the indurated " core," and the formation and discharge of healthy pus, followed by rapid granulation, and not followed by a repetition of the swellings at other points.

In those children that are, from some constitutional vice,
ibject to a constant repetition of ill-conditioned of ten greatly annoying them, and, from their very slow ten-
ond dency to heal, last for weeks and months, the sulphide of cal-
cium will soon effect a rapid recovery and prevent a tencium will soon effect a rapid recovery and prevent a ten-
dency to their return. I have recently used this remedy in a few cases of visceral
ndurations of long standing, with the effect of a rapid removal of the morbid condition and a speedy improvement in the general health. I am now trying its influence over some very intractable diseases, but can not yet speak of its power I have noticed, in the use of this agent, that there has
I been a general improvement in the nealth and vigor, and that
especially the nervous system seemed to become more effibe a general improvement in the secretions; the skin and kidneys would act more freely; the bowels, if costive, would be come at once regular; any tendency to acidity of the stomach
would be partially or completely removed. I believe its influence in toning the nervous system is an eff ect fully as impor-
tant as its alterative power. Time and an extensive use of this agent will decide whether it will maintain the high stand Ringer claims the same effect for the sulphides of potassi$\mathrm{m}$ and sodium. I have only used the sulphide of calcium. Dr. Ringer recommends doses of one tenth of a grain re-
peated every two hours. It has been my habit to give to young children a half grain every four to six hours, and to an day. Its odor is disagreeable, and similar to that given off by phuretted hydrogen. The taste, however, is easily concealed
phos by mixing with sugar or making into pill. The solution in very unpleasant to the patient to take.

I have no reason to believe the agent would have any special power over syphilitic indurations of any kind, but have
not extended the use of the agent in this direction to ascerain what power it might exert, except in a single case, where it was used in connection with mercury. The results were
good, but whether the agent had any influence or not, I can CHLORIDE OF SODA IN CANCEROUS ULCERS. By G. M. RIVERS, M.D.

ABout three years ago, I was requested to visit an old lady
with an ugly ulcer of tlet: left breast. The whole gland was with an ugly ulcer of thlt: left breast. The whole gland was
involved in the disease, hard and uneven to the touch - the ulcer three inches in diameter, quite deep, with raised, ragged and irregular edges; in fact, presenting all the appearance of
true cancer. I had previously attended two cases of cancer of
the breast, both of which presented the same appearance and the breast, both of which presented the same appearance and
proved fatal. I held out no hope of recovery, but advised and instituted, as I believed, a purely palliative treatment. I directed the ulcer to be covered with simple cerate, and, to ounces of the chloride to five of water. In three or four doses of morphine to relieve pain, the ulcer had perfectly healed, the swelling and inflammation subsided, and the pa-
tient cured. It has now been more than two years, and the The second case upon which I tried this remedy was a
The shosion to return. young negro woman, alout twenty, with half the left nostril
and a large portion of the upper lip destroyed. This woman had been to the hospital im Charleston, and the physicians
there had pronounced it cancer. Upon this case I used the chloride very freely, injecting into the nares as well as apply-
ing it externally. In four months the patient was cured, the last integument restored, leaving less delormity than I thought
possible.* In this case, also, I used no other remedy, except possible.* In this case, also, I used no other remedy, except
to protect it from the air with simple cerate. The question will naturally occur, Were these cases true tainly had no doubts when I first saw them. But may not the chloride of soda be a remedy? It is as good as any thing else. Why not try it? Some of our most useful remedies
have been discovered by accident.-Southern Medical Record.

\section{A MUltiple aNtidote.}

DR. JEANNEL, of Paris, has endeavored to answer the quescon, Is it possible to prepare an agent which shall be offineutralizing chemically all poisons in the stomach or in the relatively inoffensive, and then determining into compound uation? He discusses the applicability of the agents suggested by Dorvaul for this purpose, pointing out what appears to him to be the excell
finally proposes the following:

Solution of ferric sulphate, s. g. 1.45.

Grammes.

Calcined magnesia

Let the solution of ferric sulphate be kept in a bottle by itself, and the magnesia and charcoal mixed with the water in the other bottle, and shake violently. Thie mixture should be given repeatedly in doses of fifty to one hundred grammes. in suitable proportions, renders completely insoluble the preparations of arsenic, zinc, and digitaline, but not the oxide
of copper. It leaves in solution notable quantities of mercuric of copper. It leaves in solution notable quantities of mercuric and does not decompose or precipitate either the cyanide of
mercury or tartar emetic. It completely saturates free iodine, but acts only partially on solution of the alkaline hyTHE PRESERVATION OF ICE AT THE BEDSIDE. CuT a piece of flannel about nine inches square, and secure it by ligature round the mouth of an ordinary tumbler, so as
to leave a cup-shaped depression of flannel within the tumbler to about half its deptl. In the flannel cup so con-
structed pieces of ice may be preserved many hours-all the longer if a piece of flannel from four to five inches square be
used as a locse cover to the ice-cup. Cheap flannel, with comparatively open meshes, is preferable, as the water easily drains through it, and the ice is thus kept quite dry.
When good flannel with close texture is employed, a small hole must be made in the bottom of the flannel cup; other-
wise it holds the water and facilitates the melting of the ice, which is, nevertheless, preserved much longer than in a
naked cup or tumbler. A reserve supply outside the bedroom door can be secured by making a flannel cup, on the
plan above described, in a pitcher, and filling it with little plan above described, in a pitcher, and filling it with little
Jumps of ice, care being taken that there is space enough below the bag to allow the water to collect and leave the ice
dry. This provision will allow ice to be used during the dry. This provision will allow ice to be used during the
hottest nights without the supply failing or the patient being disturbed-two very important considerations. The real
therapeutic benefit of ice is only produced in some cases by
its free use, and its soothing and stilling effect must be aided

FELIzET, of Elbeuf having observed that in epidemics of foot-and-mouth disease no beast affected with cow-pox is ever and not one of the twenty. five beasts effectually vaccinated showed any sign or foot-and-mouth disease, even after * This women' $\mathrm{f}$ f ather died with cancer. 